\title{
Prognostic Value of Circulating Lipoprotein in Patients with Locoregionally Advanced Nasopharyngeal Carcinoma
}

\author{
Ji-Jin Yao ${ }^{\mathrm{a}, \mathrm{b}}$ Xiao-Jun He ${ }^{\mathrm{a}}$ Wayne R. Lawrence ${ }^{c}$ Wang-Jian Zhang ${ }^{\mathrm{c}}$ Jia Kou $\mathrm{Kou}^{\mathrm{a}}$ \\ Fan Zhang $^{\mathrm{b}}$ Guan-Qun Zhou ${ }^{\mathrm{a}}$ Si-Yang Wang ${ }^{\mathrm{b}}$ Ying Sun ${ }^{\mathrm{a}}$ \\ aDepartment of Radiation Oncology, Sun Yat-sen University Cancer Center; State Key Laboratory \\ of Oncology in South China, Collaborative Innovation Center for Cancer Medicine, Guangdong \\ Key Laboratory of Nasopharyngeal Carcinoma Diagnosis and Therapy, Guangzhou, Guangdong, \\ bDepartment of Radiation Oncology, the Fifth Affiliated Hospital of Sun Yat-sen University, Zhuhai, \\ Guangdong, China, 'Department of Environmental Health Sciences, School of Public Health, University \\ at Albany, State University of New York, Rensselaer, USA
}

\section{Key Words}

Nasopharyngeal carcinoma $•$ Locoregionally advanced $\bullet$ Lipoprotein $•$ Ratio $•$ Prognostic value

\begin{abstract}
Background/Aims: Lipoproteins have been reported to be associated with prognosis in various cancers; however, the prognostic value of lipoproteins in patients with nasopharyngeal carcinoma (NPC) remains largely unknown. We aim to asses the role of circulating lipoproteins in locoregionally advanced NPC patients. Methods: Between October 2009 and August 2012, a total of 1,081 patients with stage III-IVB NPC were included in the analysis. Circulating highdensity lipoprotein (HDL) and low-density lipoprotein (LDL) are the two key lipoproteins, which were measured at baseline. Receiver operating characteristic (ROC) curve analysis was used to evaluate different cut-off points for lipoproteins. Actuarial rates were performed using Kaplan-Meier methods and the log-rank test. Results: The cutoff points of HDL, LDL, and $\mathrm{LDL} / \mathrm{HDL}$ ratio were $1.17 \mathrm{mmol} / \mathrm{L}, 3.75 \mathrm{mmol} / \mathrm{L}$, and 2.73, respectively. At 5 years, high $\mathrm{HDL}$ ( $>1.17 \mathrm{mmol} / \mathrm{L}$ ) was significantly associated with better overall survival (OS, $86.6 \%$ vs. $78.9 \%$; $P=0.004$ ), distant metastasis-free survival (DMFS, $86.9 \%$ vs. $80.8 \% ; P=0.004$ ), locoregional relapse-free survival (LRFS, $90.8 \%$ vs. $85.4 \% ; P=0.010$ ), and progression-free survival (PFS, $79.1 \%$ vs. $70.2 \% ; P=0.001)$ than low $\mathrm{HDL}(\leq 1.17 \mathrm{mmol} / \mathrm{L})$. In contrast, high LDL $(>3.75 \mathrm{mmol} / \mathrm{L})$ tend to be inferior OS (79.1\% vs. 84.9\%; $P=0.016)$ in compassion with low LDL ( $\leq 3.75 \mathrm{mmol} / \mathrm{L})$. Likewise, patients with high LDL/HDL ratio (>2.73) tend to be inferior OS $(79.3 \%$ vs. $86.9 \%$; $P=0.001)$, DMFS ( $81.9 \%$ vs. $86.5 \% ; P=0.030)$, and PFS (72.6\% vs. $77.8 \% ; P=0.034)$ than those of low LDL/HDL ratio $(\leq 2.73)$. In multivariate analysis, baseline $\mathrm{HDL}$ was found to be a significant prognostic factor for LRFS (HR= 0.65; 95\% CI, 0.45-0.93; $P=0.019)$ and PFS (HR=0.75; 95\% CI,
\end{abstract}

Ji-Jin Yao and Xiao-Jun He contributed equally to this work

Ying Sun, Ph.D.

and Si-Yang Wang, M.D.
Dept. Radiation Oncology, Sun Yat-sen Univ. Cancer Center; Guangdong Key Lab. of Nasopharyngeal Carcinoma Diagnosis and Therapy, Guangzhou 510060, Guangdong, (China); Tel. +86-20-87343816, E-Mail sunying@sysucc.org.cn, 13570608929@163.com 
0.58-0.98; $P=0.034)$. Conclusions: Circulating HDL is significantly associated with treatment outcomes in patients with locoregionally advanced NPC. We suggest that HDL measurements will be of great clinical significance in the management of NPC.

\section{Introduction}

Previous studies have reported that nasopharyngeal carcinoma (NPC) is a highly prevalent malignancy in Southern China, with the annual incidence rate between 15 and 50 cases per 100, 000 persons [1]. Currently, the Tumor-Node-Metastasis (TNM) stage is mainly used to predict NPC prognosis. However, prediction of NPC patients survival still remains challenging within current TNM staging system, and patients in the same TNM stage often undergo substantial clinical heterogeneity [2]. To date, plasma Epstein-Barr viral (EBV) DNA titre remains the only biomarker with clinical utility in NPC [3, 4]. However, the high cost and the large interlaboratory variability of examination of EBV DNA enables the difficulty to apply in routine clinical practice [5]. Thus, it is of great interest to screen some inexpensive, objective, and easily detected markers to complement the TNM staging system in prognostication of NPC.

Lipoprotein plays an important role in cellular structure, as well as a precursor to several biochemical pathways [6, 7]. The two key lipoproteins are high-density lipoprotein (HDL) and low-density lipoprotein (LDL). Several studies have reported a significant inverse relation between lipoproteins and the risk of cancer at multiple sites [8-10]. Furthermore, recent studies have demonstrated that HDL was a favorable prognostic marker in several cancers, including cancers of the lung, breast and stomach [11-14]. Studies have also suggested that LDL enhances the colorectal cancer progression via reactive oxygen species (ROS) and the mitogen-activated protein kinase (MAPK) pathway [13]. For this reason, a question arises whether lipoprotein is associated with survival among patients with established NPC. However, limited studies have examined lipoprotein effects on the prognosis of NPC.

On the basis of this premise, we conducted a retrospective study to gain insight into long-term prognostic impact of lipoprotein on the outcome of patients with NPC, and explore the association between lipoproteins and clinical features from a large sample population.

\section{Materials and Methods}

\section{Ethics statement}

This study was conducted in compliance with institutional policy to protect patients' private information, and was approved by the Institutional Review Board of Sun Yat-sen University Cancer Center. The authenticity of this article has been validated by uploading the key raw data onto the Research Data Deposit (RDD) public platform, with the approval RDD number as RDDA2017000333.

\section{Study patients}

Patients were included if the following criteria were simultaneously satisfied: (1) biopsy-proven World Health Organization 2-or 3-histopathologic type NPC; (2) stage III-IVB disease based on the $8^{\text {th }}$ edition of the American Joint Committee on Cancer (AJCC) staging system [15]; (3) adequate hematological function (white blood cell counts $\geq 4.0 \times 10^{9} / \mathrm{L}$ and platelet counts $\geq 100 \times 10^{9} / \mathrm{L}$ ), adequate renal function (creatinine $[\mathrm{Cr}]<1.5$ times the normal value $<2$ times the normal values) and adequate hepatic function (total bilirubin [TBIL] and alanine aminotransferase <2 times the normal values); (4) no evidence of distant metastases. Patients were excluded if they were pregnant; previous malignancy, or had unstable cardiac disease needing treatment.

Lipoprotein measurements

All blood samples from NPC patients were obtained before any clinical treatment. Circulating HDL and LDL were measured with fasting blood samples by standard tests. 
Treatment and follow-up

All patients were treated according to the principle of treatment for NPC at our center. The prescribed doses of radiotherapy and chemotherapeutic regimens were the same as that described previously [16]. Our primary endpoint was overall survival (OS), and our secondary endpoints were distant metastasis-free survival (DMFS), locoregional relapse-free survival (LRFS), and progression-free survival (PFS). Patients during the first 2 years were seen every three months, years 3-5 every six months, and annually thereafter until death. The duration of follow-up was calculated from the first day of therapy to either the day of death or the day of the last examination.

\section{Statistical analysis}

Receiver operating characteristic (ROC) curve analysis was used to evaluate different cut-off points for lipoprotein. The area under the ROC curve was used to assess the predicted validity of lipoprotein, based on the method of Hanley and McNeil $[17,18]$. The $\chi^{2}$ test was used to evaluate the association of lipoproteins with tumor stage. Actuarial rates were performed using Kaplan-Meier methods and the log-rank test. Multivariate analyses were performed using the Cox proportional hazards model to test the independent significance of different factors. All tests were twosided, and we deemed $P$ values of less than 0.05 to be significant. All statistical analyses were completed with R 3.1.2.

\section{Results}

\section{Patient characteristics}

Between October 2009 and August 2012, a total of 1081 patients with NPC who fulfilled the inclusion criteria were included. The characteristics of the 1081 patients were presented in Table 1 . Among the 1081 patients, the median HDL was $1.27 \mathrm{mmol} / \mathrm{L}$ (Interquartile range (IQR): $1.07-1.55 \mathrm{mmol} / \mathrm{L}$ ), the median LDL was $3.25 \mathrm{mmol} / \mathrm{L}$ (IQR: $2.73-$ $3.84 \mathrm{mmol} / \mathrm{L}$ ), and the median LDL/HDL ratio was 2.66 (IQR: 2.12-3.38). The male to female ratio was 3.2:1 (826 men and 255 women), and the median age was 45 years (IQR: $37-53$ years). By TNM stage, $662(61.2 \%)$ patients were at stage III and 419 (38.8\%) at stage IVA-B. Concurrent chemoradiotherapy (CCRT) alone was delivered to 471 patients $(43.6 \%)$, and neoadjuvant chemotherapy (NACT) plus concurrent chemoradiotherapy was delivered to 610 patients $(56.4 \%)$. The median follow-up for the entire cohort was 63.6 months (range: 1.2-86 months), and the 5-year survival rates for all patients were as follows: OS, 83.1\%; DMFS, 84.5\%; LRFS, 89.0\%; and PFS, 75.1\%.
Table 1. Characteristics of 1081 patients. Abbreviation: HDL, high-density lipoprotein; LDL, low-density lipoprotein; LDL/HDL ratio, low-density-to-high-density lipoprotein ratio; CCRT, concurrent chemoradiotherapy; NACT, neoadjuvant chemotherapy

\begin{tabular}{|c|c|}
\hline Characteristic & No. of patients (\%) \\
\hline \multicolumn{2}{|l|}{ Age, yr } \\
\hline Median & 45 \\
\hline Interquartile range & $37-53$ \\
\hline \multicolumn{2}{|l|}{ Gender } \\
\hline Male & $826(76.4)$ \\
\hline Female & $255(23.6)$ \\
\hline \multicolumn{2}{|l|}{ T stage } \\
\hline $\mathrm{T} 1$ & $51(4.7)$ \\
\hline $\mathrm{T} 2$ & $80(7.4)$ \\
\hline $\mathrm{T3}$ & $676(62.5)$ \\
\hline $\mathrm{T} 4$ & $274(25.3)$ \\
\hline \multicolumn{2}{|l|}{ N stage } \\
\hline No & $95(8.8)$ \\
\hline N1 & $534(49.4)$ \\
\hline N2 & $275(25.4)$ \\
\hline N3 & $177(16.4)$ \\
\hline \multicolumn{2}{|l|}{ Overall stage } \\
\hline III & $662(61.2)$ \\
\hline IVA-B & $419(38.8)$ \\
\hline \multicolumn{2}{|l|}{ Family history } \\
\hline Yes & $292(27.0)$ \\
\hline No & 789 (73.0) \\
\hline \multicolumn{2}{|l|}{ Smoking history } \\
\hline Yes & $420(38.9)$ \\
\hline No & $633(58.6)$ \\
\hline Not available & $28(2.6)$ \\
\hline \multicolumn{2}{|l|}{ HDL (mmol/L) } \\
\hline Median & 1.27 \\
\hline Interquartile range & $1.07-1.55$ \\
\hline \multicolumn{2}{|l|}{ LDL (mmol/L) } \\
\hline Median & 3.25 \\
\hline Interquartile range & $2.73-3.84$ \\
\hline \multicolumn{2}{|l|}{ LDL/HDL ratio } \\
\hline Median & 2.66 \\
\hline Interquartile range & $2.12-3.38$ \\
\hline \multicolumn{2}{|l|}{ Chemotherapy } \\
\hline CCRT alone & $471(43.6)$ \\
\hline NACT+CCRT & $610(56.4)$ \\
\hline
\end{tabular}


The prognostic value of lipoproteins

The cut-off values for the HDL, LDL, and LDL/HDL ratio were $1.17 \mathrm{mmol} / \mathrm{L}$, $3.75 \mathrm{mmol} / \mathrm{L}$ and 2.73 , respectively, as determined by ROC curves. Based on optimal cutoff points, each biomarker was dichotomized into the high and low groups. At 5 years, high HDL was robustly associated with an improvement of OS (86.6\% vs 78.9\%; $P=0.004$ ) (Fig. $1 \mathrm{~A}$ ), DMFS (86.9\% vs $80.8 \%$; $P=0.004$ ) (Fig. 1B), LRFS (90.8\% vs $85.4 \% ; P=0.010$ ) (Fig. 1C), and PFS (79.1\% vs $70.2 \%$; $P=0.001$ ) (Fig. 1D) than low HDL. In contrast, patients with high LDL had significantly inferior oS $(79.1 \%$ vs 84.9\%; $P=0.016$ ) (Fig. 2A) than those of patients with low HDL. Although high LDL tend to be inferior DMFS (82.2\% vs $85.2 \% ; P=0.173$ ) (Fig. 2B), LRFS (88.4\% vs $88.6 \% ; P=0.813$ ) (Fig. $2 \mathrm{C}$ ), and PFS (72.5\% vs 76.4\%; $P=0.104$ ) (Fig. 2D) in comparison with low LDL, this trend did not reach statistical significance $(P>0.05$ for all). Likewise, we did not observe any difference in LRFS between patients with high and low LDL/HDL ratio (87.2\% vs. $89.8 \%, P=0.233$; Fig. 3C). However, the 5 -year OS (79.3\% vs. $86.9 \% ; P=0.001)$ (Fig. 3A), DMFS (81.9\% vs. $86.5 \%$; $P=0.030)$ (Fig. 3B) and PFS (72.6\% vs. 77.8\%; $P=0.034$ ) (Fig. 3D) for patients with high HDL were all significantly inferior compared to patients with low HDL.

Multivariate analysis was performed to further adjust for age, gender, T stage, $\mathrm{N}$ stage, family history, smoking history, chemotherapy, HDL, LDL, and LDL/HDL

Fig. 3. Kaplan-Meier survival curves for (A) overall survival, (B) distant metastasis-free survival, (C) locoregional recurrence-free survival, and (D) progression-free survival according to LDL/HDL ratio ( $\leq 2.73$ vs. > 2.73). (LDL/HDL ratio, low-density-to-high-density lipoprotein ratio).

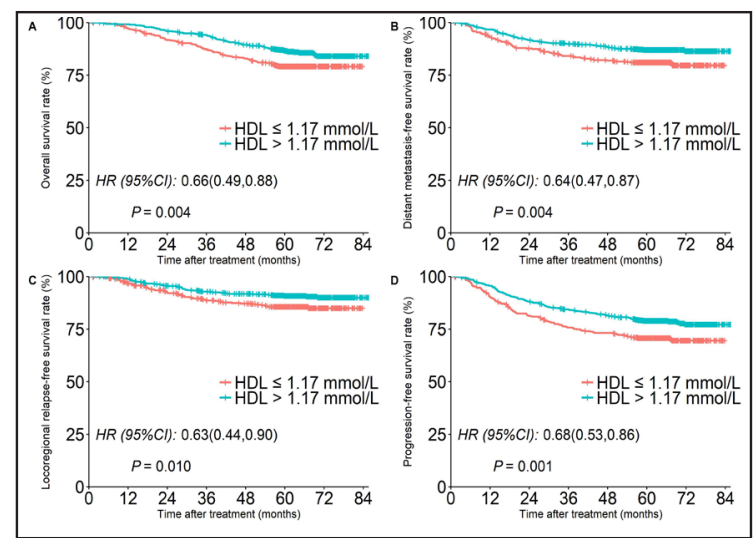

Fig. 1. Kaplan-Meier survival curves for (A) overall survival, (B) distant metastasis-free survival, (C) locoregional recurrence-free survival, and (D) progression-free survival according to circulating HDL ( $\leq 1.17 \mathrm{mmol} / \mathrm{L}$ vs. $>1.17 \mathrm{mmol} / \mathrm{L}$ ). (HDL, high-density lipoprotein).

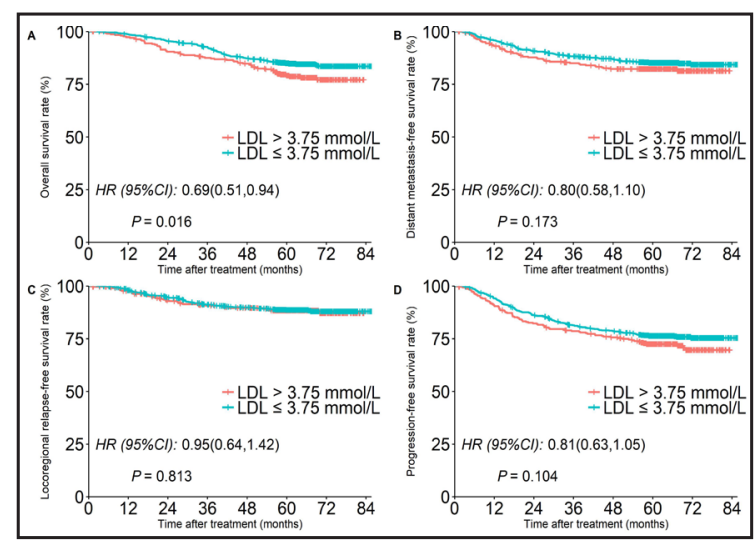

Fig. 2. Kaplan-Meier survival curves for (A) overall survival, (B) distant metastasis-free survival, (C) locoregional recurrence-free survival, and (D) progression-free survival according to circulating LDL ( $\leq 3.75 \mathrm{mmol} / \mathrm{L}$ vs. $>3.75 \mathrm{mmol} / \mathrm{L}$ ). (LDL, low-density lipoprotein).

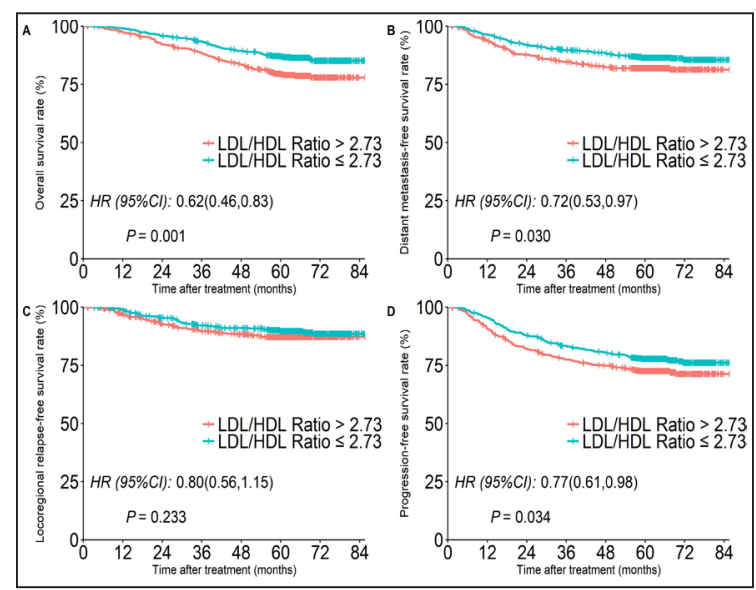


ratio. Consistent with the results of the univariate analysis, high HDL was found to be an independent favorable prognostic factor for LRFS (HR, 0.65; 95\% CI, $0.45-0.93 ; P=0.019$ ) and PFS (HR, 0.75; 95\% CI, 0.58-0.98; $P=0.034$ ) (Table 2). Additionally, these analyses revealed that age (HR, 1.55; 95\% CI, 1.15-2.09; $P=0.004)$ and $\mathrm{N}$ stage $(\mathrm{HR}, 2.24 ; 95 \% \mathrm{CI}$, 1.61-3.11; $P<0.001$ ) were independent prognostic factors for OS; N stage (HR, 2.25; 95\% CI, $1.58-3.21 ; P<0.001$ ) was independent prognostic factors for DMFS (Table 2).

\section{Correlation between HDL and clinicopathological characteristics}

In this study, ROC curve was used to evaluate different cut-off points for circulating lipoprotein. As described above, patients were divided into two groups according to HDL: high HDL ( $>1.17 \mathrm{mmol} / \mathrm{L})$ and low HDL $(\leq 1.17 \mathrm{mmol} / \mathrm{L})$. The correlations between circulating HDL and various clinicopathological features were examined (Table 3). Female patients generally had high HDL. In contrast, smokers, advanced N stage (N2/3), and stage IVA-B were more likely to present low HDL. However, no significant differences were found between two groups regarding age, T stage, family history, or chemotherapy $(P>0.05$ for all; Table 3).

Table 2. Multivariate analysis of HDL, LDL, and LDL/HDL ratio determined by ROC for patients with locoregionally advanced NPC. Abbreviation: OS, overall survival; DMFS, distant metastasis-free survival; LRFS, locoregional relapse-free survival; PFS, progression-free survival; HDL, high-density lipoprotein; LDL, low-density lipoprotein; LDL/ HDL ratio, low-density-to-high-density lipoprotein ratio; $\mathrm{HR}$, rate ratio; $\mathrm{CI}$, confidence interval

\begin{tabular}{|c|c|c|c|c|}
\hline Endpoint & Variable & HR & $95 \%$ CI for HR & $P$ value \\
\hline \multirow[t]{8}{*}{ OS } & Age & 1.55 & $1.15-2.09$ & 0.004 \\
\hline & Gender & 0.77 & $0.50-1.18$ & 0.233 \\
\hline & HDL & 0.80 & $0.57-1.12$ & 0.196 \\
\hline & LDL & 0.77 & $0.53-1.10$ & 0.149 \\
\hline & LDL/HDL ratio & 0.87 & $0.59-1.28$ & 0.473 \\
\hline & T stage & 1.18 & $0.77-1.82$ & 0.453 \\
\hline & N stage & 2.24 & $1.61-3.11$ & $<0.001$ \\
\hline & Smoking history & 3.10 & $0.97-9.94$ & 0.057 \\
\hline \multirow[t]{5}{*}{ DMFS } & Gender & 0.82 & $0.55-1.23$ & 0.341 \\
\hline & HDL & 0.81 & $0.57-1.13$ & 0.215 \\
\hline & LDL/HDL ratio & 0.87 & $0.62-1.21$ & 0.399 \\
\hline & T stage & 0.95 & $0.62-1.48$ & 0.836 \\
\hline & N stage & 2.25 & $1.58-3.21$ & $<0.001$ \\
\hline \multirow[t]{4}{*}{ LRFS } & Gender & 1.63 & $1.13-2.36$ & 0.009 \\
\hline & HDL & 0.65 & $0.45-0.93$ & 0.019 \\
\hline & T stage & 1.52 & $0.82-2.82$ & 0.185 \\
\hline & $\mathrm{N}$ stage & 1.50 & $1.01-2.23$ & 0.044 \\
\hline \multirow[t]{6}{*}{ PFS } & Age & 1.42 & $1.11-1.81$ & 0.005 \\
\hline & HDL & 0.75 & $0.58-0.98$ & 0.034 \\
\hline & LDL/HDL ratio & 0.95 & $0.73-1.23$ & 0.688 \\
\hline & T stage & 1.19 & $0.82-1.74$ & 0.359 \\
\hline & N stage & 1.81 & $1.38-2.38$ & $<0.001$ \\
\hline & Smoking history & 1.28 & $0.64-2.56$ & 0.478 \\
\hline
\end{tabular}

Table 3. Baseline characteristics of the patients with locoregionally advanced NPC stratified by HDL-C. Abbreviation: HDL, high-density lipoprotein; CCRT, concurrent chemoradiotherapy; NACT, neoadjuvant chemotherapy

\begin{tabular}{|c|c|c|c|}
\hline \multirow[b]{2}{*}{ Characteristic } & \multicolumn{2}{|c|}{ No. of patients (\%) stratified by HDL } & \multirow[b]{2}{*}{$P$ value } \\
\hline & $\leq 1.17 \mathrm{mmol} / \mathrm{L}(\mathrm{n}=410)$ & $>1.17 \mathrm{mmol} / \mathrm{L}(\mathrm{n}=671)$ & \\
\hline Age & & & 0.060 \\
\hline$\leq 45$ & $192(46.8)$ & $355(52.9)$ & \\
\hline$>45$ & $218(53.2)$ & $316(47.1)$ & \\
\hline Gender & & & $<0.001$ \\
\hline Male & $354(86.3)$ & $472(70.3)$ & \\
\hline Female & $56(13.7)$ & $199(29.7)$ & \\
\hline T stage & & & 0.705 \\
\hline T1 & $19(4.6)$ & $32(4.8)$ & \\
\hline T2 & $32(7.8)$ & $48(7.2)$ & \\
\hline T3 & $248(60.5)$ & $428(63.8)$ & \\
\hline $\mathrm{T} 4$ & $111(27.1)$ & $163(24.3)$ & \\
\hline N stage & & & 0.010 \\
\hline No & $31(7.6)$ & $64(9.5)$ & \\
\hline $\mathrm{N} 1$ & $185(45.1)$ & $349(52.0)$ & \\
\hline N2 & $109(26.6)$ & $166(24.7)$ & \\
\hline N3 & $85(20.7)$ & $92(13.7)$ & \\
\hline Overall stage & & & 0.001 \\
\hline III & $226(55.1)$ & $436(65.0)$ & \\
\hline IVA-B & $184(44.9)$ & $235(35.0)$ & \\
\hline Family history & & & 0.323 \\
\hline Yes & $118(28.8)$ & $174(25.9)$ & \\
\hline No & $292(71.2)$ & 497 (74.1) & \\
\hline Smoking history & & & $<0.001$ \\
\hline Yes & $182(44.4)$ & 238 (35.5) & \\
\hline No & $226(55.1)$ & $423(63.0)$ & \\
\hline Not available & $18(4.4)$ & $10(1.5)$ & \\
\hline Treatment strategy & & & 0.067 \\
\hline CCRT alone & $164(40.0)$ & $307(45.8)$ & \\
\hline NACT+CCRT & $246(60.0)$ & $364(54.2)$ & \\
\hline
\end{tabular}




\section{Cellular Physiology Cell Physiol Biochem 2018;48:285-292 \\ \begin{tabular}{l|l} 
DOI: 10.1159/000491728 & and Biochemistry \\
Published online: July 16, 2018 & $\begin{array}{l}\text { O 2018 The Author(s). Published by S. Karger AG, Basel } \\
\text { www.karger.com/cpb }\end{array}$
\end{tabular} \\ Yao et al.: Prognostic Value of Lipoprotein in NPC}

\section{Discussion}

According to previous studies, the prognosis of patients with NPC is far from clearly defined [2]. In the current study, we sought to determine the prognostic value of pretreatment lipoproteins in patients with locoregionally advanced NPC. Our analysis on a large sample size provided a quantitative assessment of the impact of baseline lipoproteins on the survival of locoregionally advanced NPC patients. Our findings from the present study was that circulating lipoprotein is potentially an ideal prognostic factor in patients with NPC. Serum HDL measurements will be of great clinical significance in the management of NPC.

It is well known that HDL acts as a supplier of cholesterol to tumor cells by removing excess cholesterol from peripheral tissues [19]. Several studies [11-13] have reported a significant inverse relation between HDL and disease prognosis at many sites. As shown in a study by Chi et al [11]., high HDL was an independent prognostic factor of PFS in lung adenocarcinoma patients. Another study by Wolfe et al [14]. indicated that low HDL was associated with poor OS in breast cancer patients. As an extension in the current study, high HDL was significantly associated with an improvement in the LRFS and PFS of locoregionally advanced NPC. We consider that HDL measurements will be of great clinical importance in the management of NPC, especially, when considering "decision points" in treatment algorithms.

However, Liu et al [20]. analyzed the effect of circulating HDL in NPC patients, suggesting that high HDL was an independent adverse prognostic factor in patients with NPC. There are two possible reasons for this discrepancy. One may be explained by the treatment heterogeneity from the study, since they did not account for the influence of treatment modality in their study, which might partly affect the clinical outcomes. In contrast, only patients with locoregionally advanced NPC treated with concurrent chemoradiotherapy with or without NACT were eligible for this study, which may improve the homogeneity of study group. Another possible reason is that the method of measurement in lipoprotein may partly account for the conflicting findings between Liu et al.'s study and the present study. In the study of Liu et al, majority of patients were diagnosed before 2005, and circulating HDL was measured using the antibody block method. However, with recent advances in measurement technology, fasting blood samples by standard tests were used to measure circulating HDL in the current study.

Previous studies have confirmed that circulating LDL was associated with atherosclerosis [21]. However, there remains a relative paucity of data examining the influence of circulating LDL on survival in patients with NPC. To date, only one study [22] was available for the prognostic value of serum LDL in NPC, which found that elevated LDL was associated with inferior OS. The current study also confirmed consistent significant trends between LDL and OS among patients with locoregionally advanced NPC, but this association failed to retain significance after adjusting for TNM stage. It may suggest that LDL was not the primary factor responsible for the survival in patients with locoregionally advanced NPC. As an extension in this study, we further analyzed the prognostic power of survival for LDL/ HDL ratio. Although high LDL/HDL ratio was significantly associated with inferior OS, DMFS, and PFS by univariate analyses, this association was not significant after adjusting for TNM stage. It is plausible that LDL/HDL ratio contributes to patient stratification by adding a layer of information on disease burden, which could explain in part our inability to detect a prognostic value of LDL/HDL ratio in locoregionally advanced NPC.

The associations between circulating HDL and sex have been reported before. Freedman et al [23]. reported that men tended to have lower HDL than women, which may attribute to gonadal hormones acting together with direct or indirect contributions from other sex-specific factors [24]. Consistent with the study of Freedman et al, we also found that female patients more commonly present high HDL among locoregionally advanced NPC. The present study noted a decreased HDL in cigarette smokers compared to nonsmokers, which is generally consistent with findings reported in previous studies [25, 26]. An interesting finding of this study was that patients with advanced $\mathrm{N}$ stage or stage IVA-B more often had 
low HDL. However, further investigation is required to explain the mechanism underlying this correlation between tumor stage and HDL in patients with NPC.

In summary, our results demonstrate that circulating lipoprotein is potentially an ideal prognostic factor in patients with locoregionally advanced NPC. Compared with LDL and LDL/HDL ratio, HDL might be a more reliable predictor for treatment outcomes. Thus, we consider that serum HDL measurements will be of great clinical significance in the management of NPC.

\section{Acknowledgements}

This work was supported by grants from the Health \& Medical Collaborative Innovation Project of Guangzhou City, China (201604020003), and the Special Support Program of Sun Yat-sen University Cancer Center (16zxtzlc06).

\section{Disclosure Statement}

The authors declare that they have no competing interest.

\section{References}

1 Cao SM, Simons MJ, Qian CN: The prevalence and prevention of nasopharyngeal carcinoma in China. Chin J Cancer 2011;30:114-119.

-2 Lee AWM, Ng WT, Chan LK, Chan OSH, Hung WM, Chan CC, Cheng PTC, Sze H, Lam TS, Yau TK: The strength/weakness of the AJCC/UICC staging system (7th edition) for nasopharyngeal cancer and suggestions for future improvement. Oral Oncol 2012;48:1007-1013.

-3 Wang WY, Twu CW, Chen HH, Jiang RS, Wu CT, Liang KL, Shih YT, Chen CC, Lin PJ, Liu YC, Lin JC: Longterm survival analysis of nasopharyngeal carcinoma by plasma Epstein-Barr virus DNA levels. Cancer 2013;119:963-970.

4 Lin JC, Wang WY, Chen KY, Wei YH, Liang WM, Jan JS, Jiang RS: Quantification of plasma Epstein-Barr virus DNA in patients with advanced nasopharyngeal carcinoma. N Engl J Med 2004;350:2461-2470.

5 Le QT, Zhang Q Cao H, Cheng AJ, Pinsky BA, Hong RL, Chang JT, Wang CW, Tsao KC, Lo YD, Lee N, Ang KK, Chan AT, Chan KC: An international collaboration to harmonize the quantitative plasma EpsteinBarr virus DNA assay for future biomarker-guided trials in nasopharyngeal carcinoma. Clin Cancer Res 2013;19:2208-2215.

6 Crawford ED: Understanding the epidemiology, natural history, and key pathways involved in prostate cancer. Urology 2009;73:S4-S10.

7 Heart Protection Study Collaborative Group: MRC/BHF Heart Protection Study of cholesterol lowering with simvastatin in 20, 536 high-risk individuals: A randomized placebo-controlled trial. Lancet 2002;360:7-22.

-8 Ahn J, Lim U, Weinstein SJ, Schatzkin A, Hayes RB, Virtamo J, Albanes D: Prediagnostic total and highdensity lipoprotein cholesterol and risk of cancer. Cancer Epidemiol Biomarkers Prev 2009;18:2814-2821.

-9 Jafri H, Alsheikh-Ali AA, Karas RH: Baseline and on-treatment high-density lipoprotein cholesterol and the risk of cancer in randomized controlled trials of lipid-altering therapy. J Am Coll Cardiol 2010;55:28462854.

10 Llaverias G, Danilo C, Mercier I, Daumer K, Capozza F, Williams TM, Sotgia F, Lisanti MP, Frank PG: Role of cholesterol in the development and progression of breast cancer. Am J Pathol 2011;178:402-412.

11 Chi PD, Liu W, Chen H, Zhang JP, Lin Y, Zheng X, Liu W, Dai S: High-density lipoprotein cholesterol is a favorable prognostic factor and negatively correlated with C-reactive protein level in nonsmall cell lung carcinoma. PLoS One 2014;9:e91080.

12 Guo E, Chen L, Xie Q Chen J, Tang Z, Wu Y: Serum HDL-C as a potential biomarker for nodal stages in gastric cancer. Ann Surg Oncol 2007;14:2528-2534. 


\section{Cellular Physiology Cell Physiol Biochem 2018;48:285-292

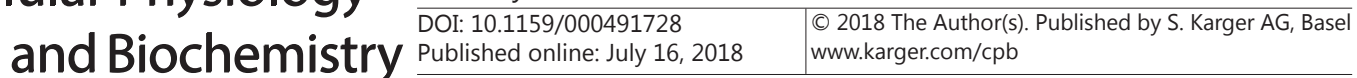

Yao et al.: Prognostic Value of Lipoprotein in NPC

13 Wang C, Li P, Xuan J, Zhu C, Liu J, Shan L, Du Q, Ren Y, Ye J: Cholesterol Enhances Colorectal Cancer Progression via ROS Elevation and MAPK Signaling Pathway Activation. Cell Physiol Biochem 2017;42:729742.

14 Wolfe AR, Atkinson RL, Reddy JP, Debeb BG, Larson R, Li L, Masuda H, Brewer T, Atkinson BJ, Brewster A, Ueno NT, Woodward WA: High-density and very-low-density lipoprotein have opposing roles in regulating tumor-initiating cells and sensitivity to radiation in inflammatory breast cancer. Int J Radiat Oncol Biol Phys 2015;91:1072-1080.

15 Amin M: American joint committee on cancer. In: AJCC Cancer Staging Manual. New York: Springer. 2016.

16 Yao JJ, Yu XL, Zhang F, Zhang WJ, Zhou GQ, Tang LL, Mao YP, Chen L, Ma J, Sun Y: Radiotherapy with neoadjuvant chemotherapy versus concurrent chemoradiotherapy for ascending-type nasopharyngeal carcinoma: a retrospective comparison of toxicity and prognosis. Chin J Cancer 2017;36:26.

17 Hanley JA, McNeil BJ: The meaning and use of the area under a receiver operating characteristic (ROC) curve. Radiology 1982;143:29-36.

18 Zweig MH, Campbell G: Receiver-operating characteristic (ROC) plots: a fundamental evaluation tool in clinical medicine. Clin Chem 1993;39:561-77.

19 Cruz PM, Mo H, McConathy WJ, Sabnis N, Lacko AG: The role of cholesterol metabolism and cholesterol transport in carcinogenesis: a review of scientific findings, relevant to future cancer therapeutics. Front Pharmacol 2013;4:119.

20 Liu YY, Lin SJ, Chen YY, Liu LN, Bao LB, Tang LQ Ou JS, Liu ZG, Chen XZ, Xu Y, Ma J, Chan AT, Chen M, Xia YF, Liu WL, Zeng YX, Mai HQ, Zeng MS, Pan JJ, Zhang X: High-density lipoprotein cholesterol as a predictor of poor survival in patients with nasopharyngeal carcinoma. Oncotarget 2016;7:42978-42987.

21 Liang C, Wang X, Hu J, Lian X, Zhu T, Zhang H, Gu N, Li J: PTPRO Promotes Oxidized Low-Density Lipoprotein Induced Oxidative Stress and Cell Apoptosis through Toll-Like Receptor 4/Nuclear Factor $\kappa \mathrm{B}$ Pathway. Cell Physiol Biochem 2017;42:495-505.

22 Tang Q, Hu QY, Piao YF, Hua YH: Correlation between pretreatment serum LDL-cholesterol levels and prognosis in nasopharyngeal carcinoma patients. Onco Targets Ther 2016;9:2585-2591.

-23 Freedman DS, Otvos JD, Jeyarajah EJ, Shalaurova I, Cupples LA, Parise H, D’Agostino RB, Wilson PW, Schaefer EJ: Sex and age differences in lipoprotein subclasses measured by nuclear magnetic resonance spectroscopy: the Framingham Study. Clin Chem 2004;50:1189-1200.

24 Wang X, Magkos F, Mittendorfer B: Sex differences in lipid and lipoprotein metabolism: it's not just about sex hormones. J Clin Endocrinol Metab 2011;96:885-893.

25 Garrison RI, Kannel WB, Feinleib M, Castelli WP, McNamara PM, Padget Si: Cigarette smoking and HDL cholesterol. The Framingham Offspring study. Atherosclerosis 1978;30:17-25.

-26 Nakamura K, Barzi F, Huxley R, Lam TH, Suh I, Woo J, Kim HC, Feigin VL, Gu D, Woodward M: Does cigarette smoking exacerbate the effect of total cholesterol and high-density lipoprotein cholesterol on the risk of cardiovascular diseases? Heart 2009;95:909-916. 